\title{
Microphase structuring in two-dimensional magnetic colloid mixtures
}

\author{
Norman Hoffmann, Christos N Likos ${ }^{1}$ and Hartmut Löwen \\ Institut für Theoretische Physik II: Weiche Materie, Heinrich-Heine-Universität Düsseldorf, \\ Universitätsstraße 1, D-40225 Düsseldorf, Germany \\ E-mail: likos@thphy.uni-duesseldorf.de
}

Received 29 August 2006, in final form 3 October 2006

Published 26 October 2006

Online at stacks.iop.org/JPhysCM/18/10193

\begin{abstract}
Suspensions of super-paramagnetic colloids trapped in the water-air interface are an excellent model system for the study of two-dimensional systems. Here, we investigate the structural behaviour of two-component mixtures of such particles. The interparticle interactions are tunable through the application of a magnetic field perpendicular to the air-water surface. Further, they can be influenced by the choice of the relative magnetic susceptibilities of the two colloidal species. We have performed integral equation theory and computer simulations to study the static properties of such a binary superparamagnetic system. For all susceptibility ratios studied, no macroscopic phase separation takes place; the fluid phase remains macroscopically homogeneous but microphase structuring occurs: the interactions with the large particles lead to a clustering of the smaller particles. We combine structural information in reciprocal space together with morphological measures in real space to characterize the ordering of the two species in the binary mixture.
\end{abstract}

\section{Introduction}

Super-paramagnetic colloidal particles that are confined by gravity on the two-dimensional water-air interface of a pendant droplet are excellent realizations of strictly two-dimensional (2D) classical many-body systems; references [1,2] provide recent reviews on the new physics in these 2D systems. An external magnetic field, directed perpendicularly to the droplet surface, induces magnetic moments on these particles that align themselves with the applied field. Due to the super-paramagnetic character of the particles, thermal fluctuations of the magnetic moment around the preferred direction are negligible; thus the magnetic moments of the particles are fixed in the direction prescribed by the external field. The strength of the induced dipole moment, on the other hand, can conveniently be tuned by the magnitude of the

1 Author to whom any correspondence should be addressed. 
external magnetic field. This leads to an effective repulsion between the spheres through their induced parallel dipole moments.

While a one-component system of almost monodisperse particles has been extensively studied both for the fluid and for the crystalline phases [3-13], only recently have twocomponent mixtures of such systems been investigated [14-16]. Of particular interest here is to tune, via control of the susceptibility asymmetry, the so-called non-additivity of the mutual interaction potentials between the species of the mixture. For positive non-additivity, macroscopic phase separation may take place $[17,18]$ whereas, for negative values of the same, microphase separation and clustering of the particle species in domains within the fluid might occur-qualitatively similar to results obtained in three spatial dimensions and effective onecomponent systems [19-25]. A wide variety of systems displaying circular and striped pattern formation has been analysed, such as magnetic garnets, Langmuir monolayers and thin films of adsorbates [26, 27]. Stable cluster formation has been observed in systems interacting via the competition between a short-ranged attraction and a long-ranged repulsion [28] and it can occur even in the complete absence of attractions, provided that the repulsive potential is bounded and decays sufficiently rapidly to zero at large interparticle distances [29]. The competition between a short-ranged Lennard-Jones-like and a long-ranged repulsive Yukawa potential leads to a cluster formation that has been proved by ground-state energy calculations [30]. This system even exhibits a gel phase driven by the inherent self-generated cluster polydispersity [31]. Also nanoparticles deposited at the air-water interface show spontaneous patterning in a cluster-like structure [32].

In this work, we investigate the liquid structure and local ordering in a $2 \mathrm{D}$ binary mixture interacting via dipole-dipole repulsions using integral equation theories and computer simulations that compare very well with each other and with previously published experimental results [16]. Macrophase separation (demixing) does not take place for any combination of physical parameters, a property that can be traced back to the fact that the non-additivity parameter of the interactions is negative irrespective of the value of the magnetic susceptibility ratio. Instead, microphase structuring with a partial clustering of the weaker species takes place, with a sponge-like structure emerging. Partial structure factors and a morphological characterization of the particle positions obtained in computer simulations and employing the Euler characteristic $[33,34]$ are used to characterize the ordering of the two species in the uniform mixture. This partial clustering has been explicitly confirmed experimentally [16]; in this work, we present a detailed analysis of the microphase structuring for a wide spectrum of relevant physical parameters.

The rest of this paper is organized as follows. In section 2 we define the system and the associated interparticle interactions. The integral equation theory is concisely presented in section 3. Results for the partial structure factors are presented in section 4 and the morphological analysis in section 5. Finally, in section 6 we summarize and draw our conclusions. Some technical details on fast two-dimensional Fourier transforms are relegated to appendix A and mathematical material on the derivation of the Euler characteristic on a square lattice is presented in appendix B.

\section{The systems and the interaction potentials}

\subsection{One-component system}

We consider a two-dimensional system of $N$ colloidal particles in a plane of surface area $A$. With $\rho=N / A$ denoting the area density of the particles, we introduce the average interparticle distance $a \equiv 1 / \sqrt{\rho}$ as a characteristic length scale of the system. The motion of the particles 
is confined by a water-air interface. We expose the system to an external static magnetic field $\mathbf{B}_{\mathbf{0}}$ that induces on each particle a magnetic moment $\mathbf{m}$. We restrict ourselves to the case of a magnetic field pointing perpendicular to the plane. The particles are assumed to be superparamagnetic, i.e., the magnetic dipole of each particle aligns perfectly with the external field. Thus, it holds that

$$
\mathbf{m}=\chi \mathbf{B}_{\mathbf{0}},
$$

where the proportionality constant $\chi$ is the particles' magnetic susceptibility. For the system at hand, the dipole-dipole interaction between two particles with parallel dipole moments $\mathbf{m}_{i}=\chi \mathbf{B}_{\mathbf{0}}$ and coordinates $\mathbf{r}_{i}, i=1,2$, reads as $[10,35]^{2}$

$$
u\left(\mathbf{r}_{1}, \mathbf{r}_{2}\right)=\frac{\left(\chi B_{0}\right)^{2}}{\left|\mathbf{r}_{1}-\mathbf{r}_{2}\right|^{3}}\left(1-3 \sin ^{2} \omega \cos ^{2} \theta\right)
$$

where $\omega$ is the angle between the field and the vertical on the confining plane and $\theta$ the angle between $\mathbf{m}_{i}$ and the direction of the connecting vector, $\mathbf{r}_{1}-\mathbf{r}_{2}$. For the case at hand (perpendicular field), we have $\omega=0^{\circ}$ and $\theta=90^{\circ}$; thus equation (2) simplifies into

$$
u(r)=\frac{\left(\chi B_{0}\right)^{2}}{r^{3}}
$$

where $r=\left|\mathbf{r}_{1}-\mathbf{r}_{2}\right|$. For the purely repulsive potential at hand, the system is thermodynamically stable on the basis of the dipole-dipole interaction alone; an additional, short-range repulsion is not necessary to stabilize the system at the relevant experimental densities, as the typical interparticle distances are large enough (compared to their physical size) and only the dipoledipole interaction is felt ${ }^{3}$. Comparisons with experimental results $[12,16]$ fully support the above modelling of the interactions and confirm its realistic character. The particles are thus treated as point-like. Using the aforementioned length scale $a$, we introduce the dimensionless coupling strength $\Gamma$, defined as

$$
\Gamma=\beta \frac{\left(\chi B_{0}\right)^{2}}{a^{3}},
$$

where $\beta=1 /\left(k_{\mathrm{B}} T\right)$, with $k_{\mathrm{B}}$ being Boltzmann's constant and $T$ being the temperature. Clearly, $\Gamma$ expresses the ratio of the kinetic versus typical potential energy of a particle and allows for the distinction of the weak $(\Gamma \lesssim 1)$, intermediate $(\Gamma \cong 1)$ and strong $(\Gamma>1)$ regimes. Rescaling the particle coordinates with the interparticle distance, $r_{i} \rightarrow x_{i} \equiv r_{i} / a$, and using equations (1) and (4), we can write the interaction potential, equation (3), in the form

$$
\beta u(x)=\frac{\Gamma}{x^{3}},
$$

with $x=\left|\mathbf{x}_{1}-\mathbf{x}_{2}\right|$. All structural and thermodynamic properties of such a system depend solely on the coupling strength $\Gamma$ and not separately on temperature and density.

\subsection{Two-component system}

Generalizing the ideas above, we now allow for a second component to be present in the system. Each component is characterized by its partial density $\rho_{1}, \rho_{2}$ and its susceptibility $\chi_{1}, \chi_{2}$, respectively. In the experimental setup, the particles acquire their super-paramagnetic

2 Equation (2) above holds for two permanent magnetic dipoles. For two induced magnetic dipoles, the expression must be multiplied by a factor $1 / 2$. We ignore this factor because it only leads to a trivial rescaling of the coupling strength $\Gamma$; see, e.g., [10].

3 The argument applies as long as the field does not deviate from the vertical direction by more than, roughly, $30^{\circ}$. In contrast, dipole-dipole attractions that lead to chain formation arise and a short-range steric repulsion is necessary to stabilize the system [10]. 
character through doping with $\mathrm{Fe}_{2} \mathrm{O}_{3}$. For a given doping density, the bigger the particle is, the larger its susceptibility. Therefore, in what follows, we refer to the particles having the larger susceptibility as the 'big' particles, although the physical size of the colloids does not enter in the pair potentials. Accordingly, we are now dealing with three different interactions:

$$
\beta u_{i j}(x)=\frac{\Gamma_{i j}}{x^{3}}, \quad i, j=1,2 .
$$

In equation (6) above, $x$ stands for the distance between any two particles scaled over the average interparticle distance between big particles, $x \equiv r / a_{11}$, where $a_{11}=1 / \sqrt{\rho_{1}}$. Therefore, the expressions for the interaction strengths $\Gamma_{i j}$ are generalized to

$$
\Gamma_{i j}=\frac{\beta \chi_{i} \chi_{j} B_{0}^{2}}{a_{11}^{3}}, \quad i, j=1,2 .
$$

The two-component system is completely characterized by three quantities: the density ratio $\rho_{2} / \rho_{1}$, the susceptibility ratio $\chi_{2} / \chi_{1}$ and one of the three interaction strengths, which we pick as $\Gamma_{11}$ in what follows.

\section{Integral equation theory}

\subsection{One-component system}

The determination of the pair structure of a uniform fluid amounts to the calculation of the radial distribution function (rdf) $g(r)$ and the direct correlation function (dcf) $c(r)$. By introducing the total correlation function $h(r)=g(r)-1$, the former two quantities are connected via the Ornstein-Zernike (OZ) relation [37]

$$
h(r)=c(r)+\rho \int \mathrm{d}^{d} r^{\prime} c\left(\left|\mathbf{r}-\mathbf{r}^{\prime}\right|\right) h\left(r^{\prime}\right),
$$

with the dimension of the system $d=2$ for the case at hand. The OZ relation is exact and couples two unknown functions, $h(r)$ and $c(r)$, via an integral equation. A second equation is required in order to determine these. The total correlation function can be expressed in terms of the direct correlation function as

$$
h(r)=\exp [-\beta u(r)+h(r)-c(r)+B(r)]-1,
$$

where $u(r)$ denotes the pair potential and $B(r)$ is the so-called bridge function [37]. By introducing certain approximations for the bridge function, an approximate equation to the $\mathrm{OZ}$ relation, called closure, can be supplied. The system is now fully determined and both the rdf as well as the dcf can be obtained by solving the two coupled equations numerically. By setting the bridge function equal to zero, we obtain the hypernetted chain (HNC) closure, which has proved to yield good results for long-ranged pair potentials in three dimensions. The PercusYevick (PY) closure arises by choosing the following expression for the bridge function:

$$
B_{\mathrm{PY}}(r)=-[h(r)+1-c(r)]+1+\ln [h(r)+1-c(r)] .
$$

The PY closure furnishes accurate results for short-range interactions (such as the hard-sphere potential) in three dimensions.

Once $h(r)$ is known, another interesting and important quantity to describe the structure of the system is the structure factor $S(k)$. It can be expressed simply by means of the Fourier transform of the total correlation function $\tilde{h}(k)$ :

$$
S(k)=1+\rho \tilde{h}(k) \text {. }
$$

The structure factor can be directly measured by scattering techniques, and is therefore a quantity of paramount importance for comparing theory and experiment. 


\subsection{Two-component system}

The extension from the one-component system to the multicomponent system is straightforward. Consider a two-component system consisting of $N_{1}, N_{2}$ particles enclosed in a macroscopic area $A$ and described by the three pair interactions $u_{i j}(r), i, j=1,2, r$ denoting the distance between any two particles. The pair structure is fully determined by three correlation functions $h_{i j}(r), i \leqslant j=1,2$; due to symmetry, $h_{i j}(r)=h_{j i}(r)$. The generalization of the OZ relation for multicomponent mixtures reads as

$$
\tilde{\mathbf{H}}(k)=\tilde{\mathbf{C}}(k)+\tilde{\mathbf{C}}(k) \cdot \tilde{\mathbf{D}} \cdot \tilde{\mathbf{H}}(k),
$$

where $\tilde{\mathbf{H}}(k)$ and $\tilde{\mathbf{C}}(k)$ are $2 \times 2$ symmetric matrices with elements

$$
[\tilde{\mathbf{H}}(k)]_{i j}=\tilde{h}_{i j}(k) \quad \text { and } \quad[\tilde{\mathbf{C}}(k)]_{i j}=\tilde{c}_{i j}(k),
$$

and $\tilde{\mathbf{D}}$ is a $2 \times 2$ diagonal matrix of the partial densities,

$$
[\tilde{\mathbf{D}}]_{i j}=\rho_{i} \delta_{i j} .
$$

The linear algebraic system (12) provides three independent equations with six unknown functions $\tilde{h}_{i j}(k)$ and $\tilde{c}_{i j}(k)$. Therefore, three closure relations have to be supplied to determine the unknown functions. Apart from the generalizations of the aforementioned HNC and PY closures, a particularly reliable relation is provided by the Rogers-Young closure [36] that 'interpolates' between the two and has the form

$$
h_{i j}(r)=\exp \left[-\beta u_{i j}(r)\right]\left[1+\frac{\exp \left[\gamma_{i j}(r) f_{i j}(r)\right]-1}{f_{i j}(r)}\right]-1,
$$

where $\gamma_{i j}(r)=h_{i j}(r)-c_{i j}(r)$ and $f_{i j}(r)=1-\exp \left(-\alpha_{i j} r\right)$ and the 'mixing functions' $f_{i j}(r)$ include tunable parameters $\alpha_{i j}$ that are chosen in order to enforce thermodynamic consistency. In this work, we employ a single self-consistency parameter $\alpha \equiv \alpha_{i j}$. This turns to be sufficient to bring about agreement with simulation and experiment and it guarantees the equality between the total virial and fluctuation compressibilities ${ }^{4}$. The three partial structure factors for the two component system read as

$$
S_{i j}(k)=\delta_{i j}+\sqrt{\rho_{i} \rho_{j}} \tilde{h}_{i j}(k) .
$$

In solving the two coupled integral equations, the OZ relation and the closure, one has to invoke an iterative procedure that requires the repeated calculation of convolution integrals. These are evaluated most efficiently and rapidly in reciprocal space, by making use of the tool of fast Fourier transforms. Although this procedure is straightforward in three dimensions, in which the Fourier transform of a spherosymmetric function takes the form of a one-dimensional sine transformation, in two dimensions things are more complicated. We have circumvented this problem by applying the procedure of Caillol et al [38]. A short account of this technique is given in appendix A.

\section{Correlation functions}

In this section, we present results for the structure for the one-component as well as for the twocomponent two-dimensional system of super-paramagnetic colloids exposed to a perpendicular, static external magnetic field. To corroborate the (approximate) results of the numerical solutions of the liquid integral equation theory derived in section 3, we also have performed extensive Brownian dynamics (BD) computer simulations [42]. We employed a square box with periodic boundary conditions ${ }^{5}$. We varied the size of the simulation box in order to check

\footnotetext{
4 An alternative approach is to use all three $\alpha_{i j}$ s to match the partial compressibilities also [39].

5 We stress that the dipole-dipole interaction in two dimensions is integrable and this is why we do not have to use more sophisticated schemes to treat this interaction, as is common in three dimensions [40].
} 

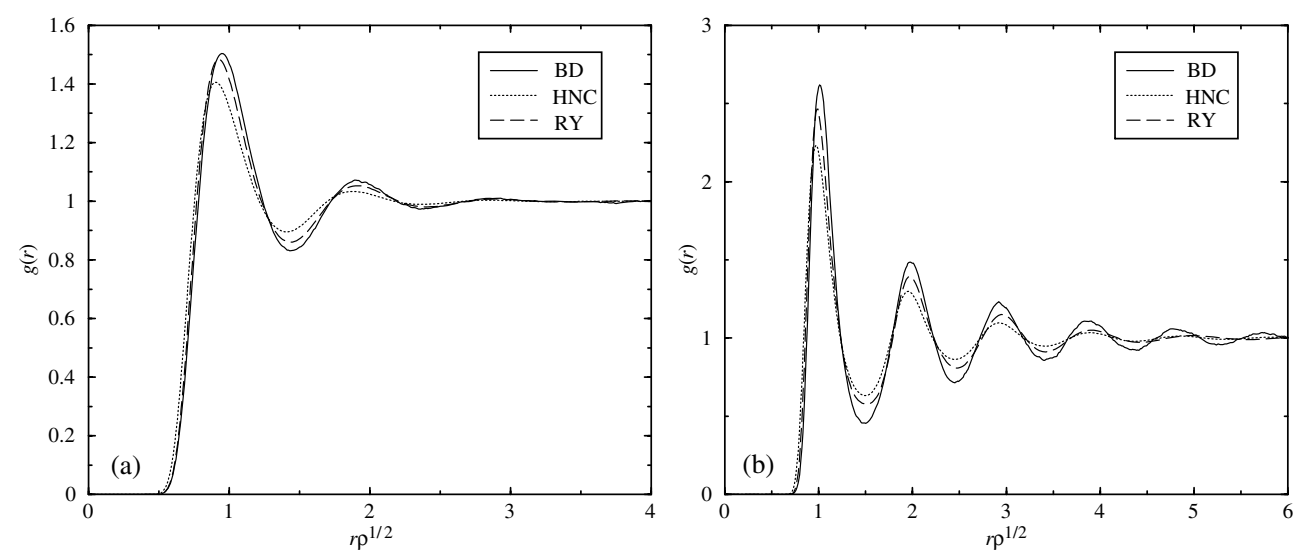

Figure 1. The radial distribution functions for the one-component system at (a) $\Gamma=1.44$ and (b) $\Gamma=8.2$. The Brownian dynamics (BD) results are compared to the hypernetted chain (HNC) and Rogers-Young (RY) closures.

carefully for finite-size effects. Typically, we found about 2000 particles to be sufficient for the latter to be negligible. The timestep for the integration of the overdamped Langevin equations of motion was $\Delta t=10^{-4} \rho D$, where $D$ is the short-time diffusion constant of the particles. A total of 10000 timesteps were used to equilibrate the system, and during a further $10^{6}$ timesteps the statistics were gathered.

\subsection{One-component system}

In figure 1, typical results for the $g(r)$ at strong coupling, $\Gamma=1.44$ and $\Gamma=8.2$, are shown. It is somewhat surprising that the agreement between HNC and BD is rather poor; based on experience for long-range interactions in three dimensions, one might have expected a better agreement. Apparently, space dimensionality is relevant. As the HNC implicitly contains elements of a 'mean-field picture' [43], it is plausible to assert that its quality worsens as $d$ diminishes. The Rogers-Young closure provides much better agreement, although also in this case the amount of correlation is slightly underestimated. The agreement between the RY closure and the simulation worsens somewhat as the coupling grows but it should be kept in mind that at $\Gamma \cong 10$ the system crystallizes [13]; thus for $\Gamma=8.2$ we are already dealing with a strongly correlated fluid. As far as experiments are concerned, we refer the reader to [41], where nearly identical results for $g(r)$ have been obtained by video microscopy.

\subsection{Two-component system}

For the rest of this work, we turn our attention to the much richer two-component case. For all tested parameter combinations, the RY closure almost perfectly matches the structural correlations predicted by the simulations. As a representative result, we provide one parameter combination in figure 2: it shows excellent agreement between the simulation data and the RY closure whereas, as could be anticipated from the one-component case, the HNC fails to be as accurate. Excellent agreement with experimental structural data for a different parameter combination has been reported in [16]. Therefore, in what follows, we rely on the RY closure to analyse the pair structure of the fluid regime. We vary the relevant system parameters, $\Gamma_{11}$, $\rho_{2} / \rho_{1}$ and $\chi_{2} / \chi_{1}$, and discuss the concomitant effects on the structural correlations. 

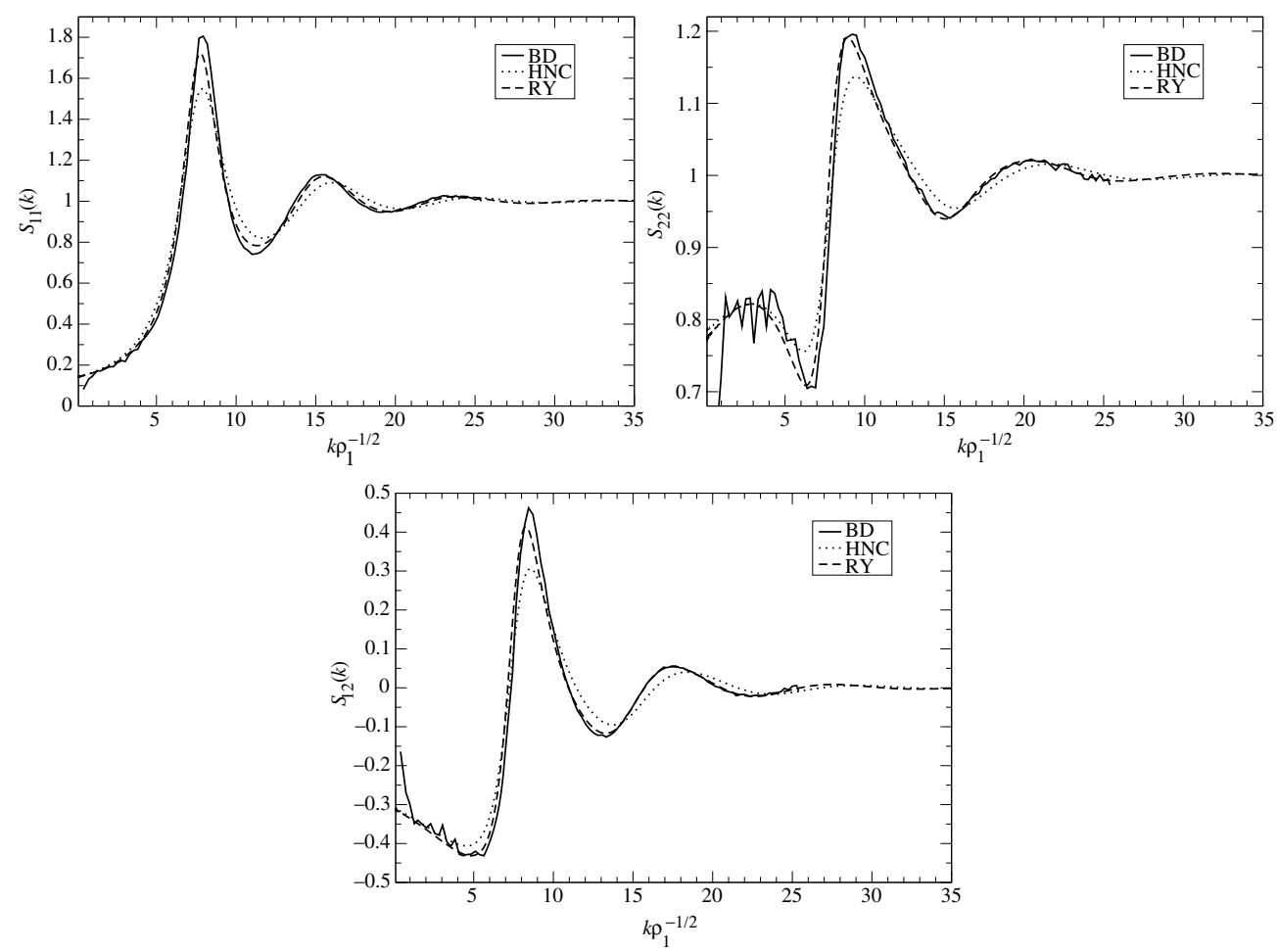

Figure 2. The partial structure factors $S_{11}(k), S_{22}(k)$, and $S_{12}(k)$ for the two-component system. Computer simulation results obtained by Brownian dynamics (BD) are compared to the HNC and RY closure. The parameter combinations here read as $\Gamma_{11}=3.0, \rho_{2} / \rho_{1}=0.5$ and $\chi_{2} / \chi_{1}=0.5$.

Let us first increase the coupling strength, covering a range from $\Gamma_{11}=1.0$ to 9.0. Results for the radial distributions functions $g_{i j}(r)$ are shown in figure 3; for all these values, the system remains fluid. At the same time, we keep the density and susceptibility ratio fixed at $\rho_{2} / \rho_{1}=0.5$ and $\chi_{2} / \chi_{1}=0.5$. For increasing interaction strength, the height of the main peak of all three distribution increases steadily, whereas the peak position $r$, measured in units of $a_{11}$, does not shift. The result is physically reasonable: we can assume that $\Gamma_{11}$ is increased by making the external field $\mathbf{B}_{0}$ stronger but otherwise leaving the particle numbers untouched. Then, the typical interparticle distances within the first coordination shell remain unchanged and only the degree of local ordering is affected, due to the increasingly strong repulsions. Note also that the extent of the 'correlation holes' $b_{i j}$, i.e., the small- $r$ region for which $g_{i j}(r) \cong 0$, differs between the three distribution functions. Indeed, the ordering $u_{22}(r)<u_{12}(r)<u_{11}(r)$ leads to the inequalities $b_{22}<b_{12}<b_{11}$.

These features are reflected in the structure factors, shown in figure 4. In reciprocal space, the height of the principal peak position grows as the coupling increases. At the same time, the structure factors reveal a striking feature that cannot be distinguished by looking at the realspace distribution functions alone: the structure factor $S_{22}(k)$ shows a pre-peak at, roughly, $k_{\text {clus }} / \sqrt{\rho_{1}} \cong 3.5$ that precedes the main peak, located at $k_{\text {part }} / \sqrt{\rho_{1}} \cong 8.5$. The latter signals ordering with a length scale $\lambda_{\text {part }}=2 \pi /\left(8.5 \sqrt{\rho_{1}}\right) \cong 0.5 / \sqrt{\rho_{2}}$, whereas the former points to the existence in the system of spatial structures formed by the small particles that have a larger typical length scale $\lambda_{\text {clus }} \stackrel{\sim}{=} 1.27 / \sqrt{\rho_{2}}$. Note that $\lambda_{\text {part }}$ is smaller than the typical interparticle distance $a_{22}=1 / \sqrt{\rho_{2}}$ between the small particles by about a factor 2 . This 

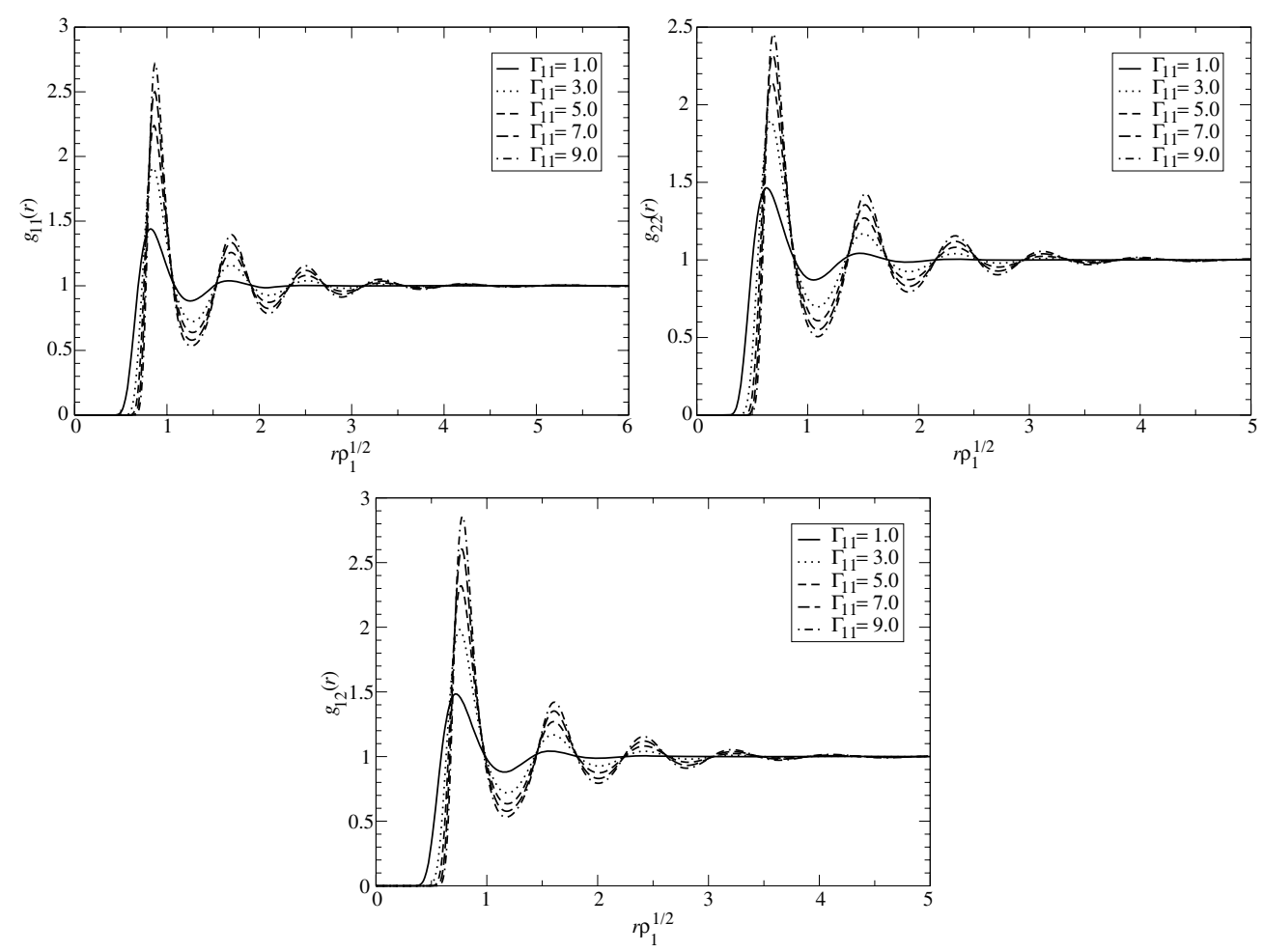

Figure 3. Partial radial distribution functions for the two-component system. Varying the interaction strength $\Gamma_{11}$, we keep the other parameters fixed: $\rho_{2} / \rho_{1}=0.5$ and $\chi_{2} / \chi_{1}=0.5$.

implies that the latter are 'squeezed together' due to the influence of the big ones. Thus, $\lambda_{\text {part }}$ corresponds to the small-small interparticle distance, whereas $\lambda_{\text {clus }}>\lambda_{\text {part }}$ describes, as will be explicitly demonstrated shortly, the extent of clusters of tightly squeezed small particles, nested in the voids formed by the big ones. The fact that the $k_{\text {clus }}$-pre-peak height is not particularly prominent points to rather diffuse clusters with an ill-defined boundary. Yet, their presence is clearly signalled by the pre-peak, whose position (and hence the cluster size) is insensitive to the coupling. We emphasize here that this is a novel phenomenon, which persists for a wide range of parameter combinations. Of particular importance is the absence of any similar feature in the big-big structure factor $S_{11}(k)$ and in the cross-structure factor $S_{12}(k)$. The physical origin of this partial clustering can be traced to the particular form of the interactions. A moderate amount of negative non-additivity (see below) as well as the ordering $u_{22}(r)<u_{12}(r)<u_{11}(r)$ is necessary, which explains the fact that partial clustering is absent both for additive hard-sphere mixtures [44] and for the positively non-additive AsakuraOosawa model [45], to mention some popular models of liquid-state and soft matter theory.

Let us then investigate the evolution of the structural characteristics when the other two free parameters of the mixture are varied. In order to examine the influence of the composition, we keep the coupling constant fixed at $\Gamma_{11}=3.0$ and the susceptibility ratio at $\chi_{2} / \chi_{1}=0.7$. Subsequently, we vary the density ratio starting from a majority of big particles and ending up with a majority of the small one. Results for the structure factors are shown in figure 5 . The height of the main peak of the structure factor remains roughly unchanged for the big particles; however, its position moves clearly to the right as small particles are added. This points to a 

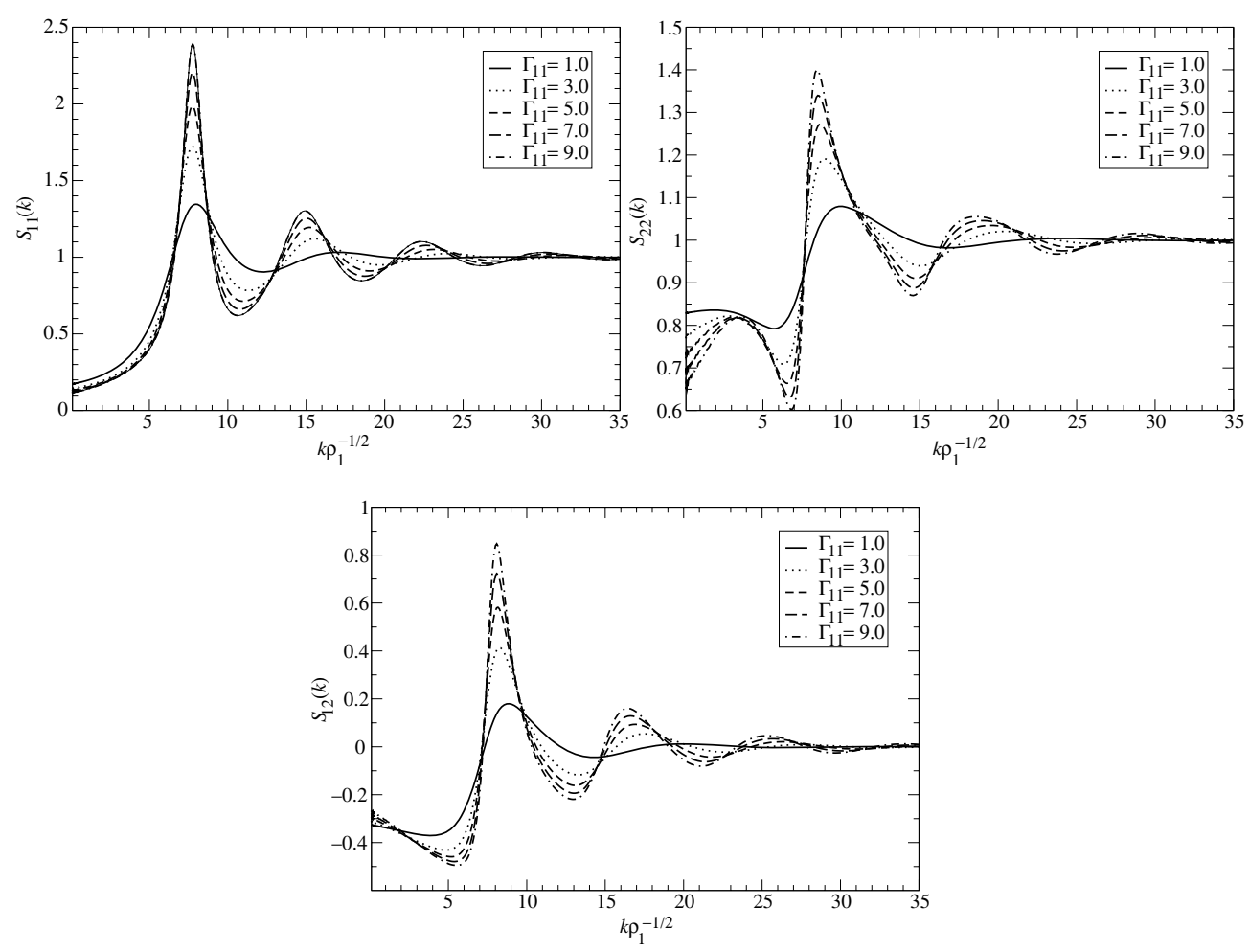

Figure 4. The partial structure factors $S_{11}(k), S_{22}(k)$, and $S_{12}(k)$ for the two-component system. The interaction strength $\Gamma_{11}$ is varied whereas the other parameters are kept fixed: $\rho_{2} / \rho_{1}=0.5$, $\chi_{2} / \chi_{1}=0.5$.

tendency of the big particles to move closer upon addition of the small ones, a feature explicitly confirmed by the evolution of their partial distribution function $g_{11}(r)$, shown in figure 6 . The small particles require space for themselves and are thus 'pushing' the big ones closer to each other; the interaction $u_{11}(r)$ is becoming effectively softer, a feature also confirmed by the increase in the partial compressibility $\chi_{1}=S_{11}(k=0) /\left(k_{\mathrm{B}} T \rho_{1}\right)$; see figure 5. However, this partial compressibility never diverges; thus the system remains mixed. Therefore, the reordering of the big particles in the presence of the small ones is more subtle: big particles are coming closer together and they open up in this way space for the small ones. The two regions of space, however, are closely intertwined with each other, as no macrophase separation takes place.

Let us now turn our attention to $S_{22}(k)$ shown in figure 5. The wandering of the "particle peak' $k_{\text {part }}$ at higher $k$-value upon increasing $\rho_{2}$ simply describes the decreasing interparticle separation between the small particles as their population grows. The 'cluster pre-peak' $k_{\text {clus }}$ is also present, albeit weak, and its position remains unchanged as $\rho_{2}$ grows. The physical interpretation is that more and more small particles are squeezed into the voids left by the big ones but the size of these voids (small-particle clusters) is insensitive to $\rho_{2}$. Note that for $\rho_{2} / \rho_{1}=0.1$ both the cluster pre-peak and the particle peak are weak, due to the low partial concentration. However, the cluster pre-peak survives also when the small particles are in the majority.

Finally, let us fix the coupling strength and the density ratio at $\Gamma_{11}=3.0$ and $\rho_{2} / \rho_{1}=0.5$ and vary the susceptibility ratio. The corresponding structure factors can be seen in figure 7 . 

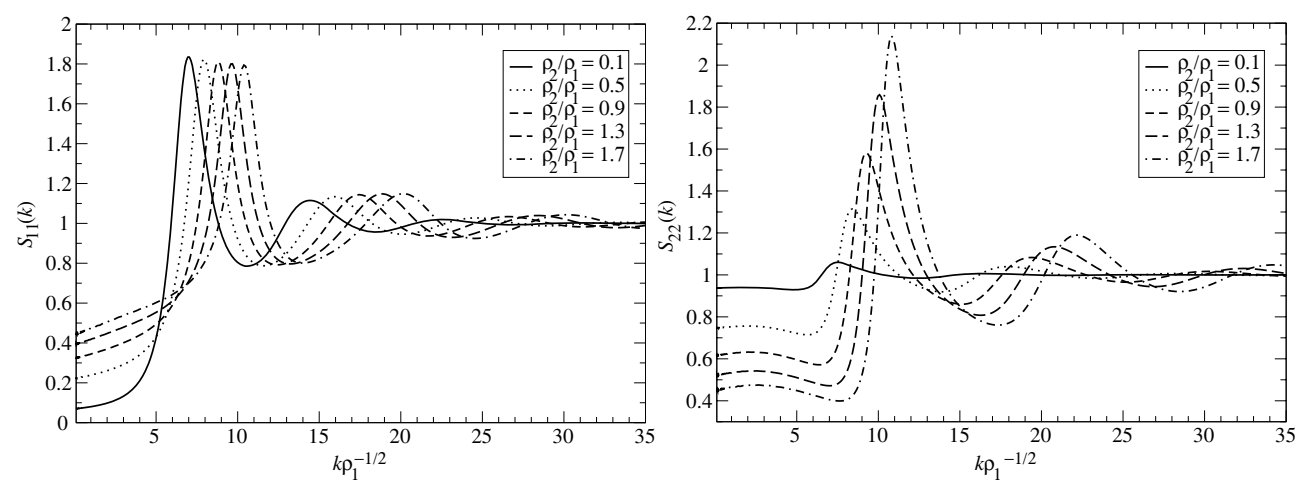

Figure 5. Partial structure factors for the two-component system. Here the density ratio is varied and the other two parameters are fixed at $\Gamma_{11}=3.0$ and $\chi_{2} / \chi_{1}=0.7$.

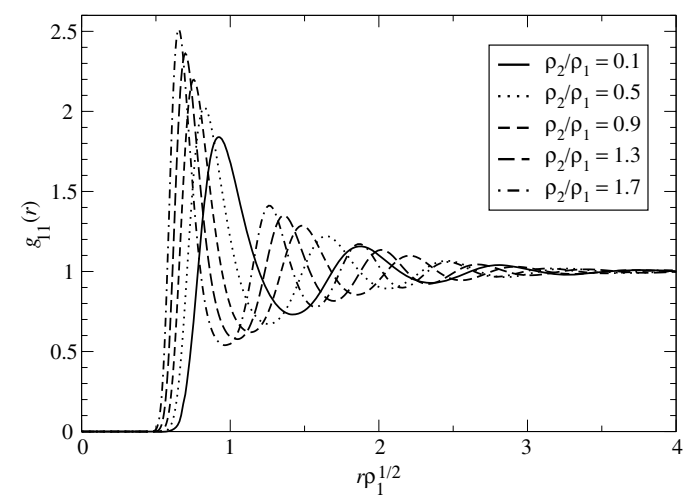

Figure 6. The radial distribution function $g_{11}(r)$ of the big particles for the parameter combination of figure 5 .

For all investigated parameter combinations, the partial structure factors remain finite for $k=0$; therefore a fluid-fluid demixing is not expected for the system at hand. Again, however, the cluster pre-peak in the structure factor $S_{22}(k)$ is visible, whereas no such signature appears in $S_{11}(k)$. Note that for low susceptibility ratio, $\chi_{2} / \chi_{1}=0.1$, the pre-peak is at least as pronounced as the 'main' peak: as the direct interaction scales as $\chi_{2}^{2}$ but the cross-interaction as $\chi_{1} \chi_{2}$, the particle-particle correlations are weaker than the cluster ones. At the other extreme, $\chi_{2} / \chi_{1}=0.7$, the pre-peak is still present but is clearly subdominant to the main peak. Indeed, there are two trivial limits for the two-component system, when it is looked upon through its dependence on the susceptibility ratio $\xi \equiv \chi_{2} / \chi_{1}$, where $0 \leqslant \xi \leqslant 1$. At $\xi=0$, the small particles are ideal and experience no forces, neither among themselves nor with the big particles. In this case, $S_{22}(k)=1$ and we are dealing with a one-component system at $\Gamma=\Gamma_{11}$. At the other extreme, $\xi=1$, the two species become identical and there is just one structure factor at a rescaled coupling parameter $\Gamma=\Gamma_{11}(1+\alpha)^{3 / 2}$, where $\alpha \equiv \rho_{2} / \rho_{1}$. Also in this case there is no subclustering phenomenon. We expect, therefore, that the pre-peak in $S_{22}(k)$ exists in some domain $\xi \in\left[\xi_{1}, \xi_{2}\right] \subset[0,1]$. We were able to show that $\xi_{1}$ is at least as small as 0.1 and $\xi_{2}$ at least as large as 0.7 .

A key quantity in understanding the phenomena at hand is the so-called non-additivity parameter $\Delta$, which can be derived from the interaction potentials $u_{i j}(r)$ as follows. First, 

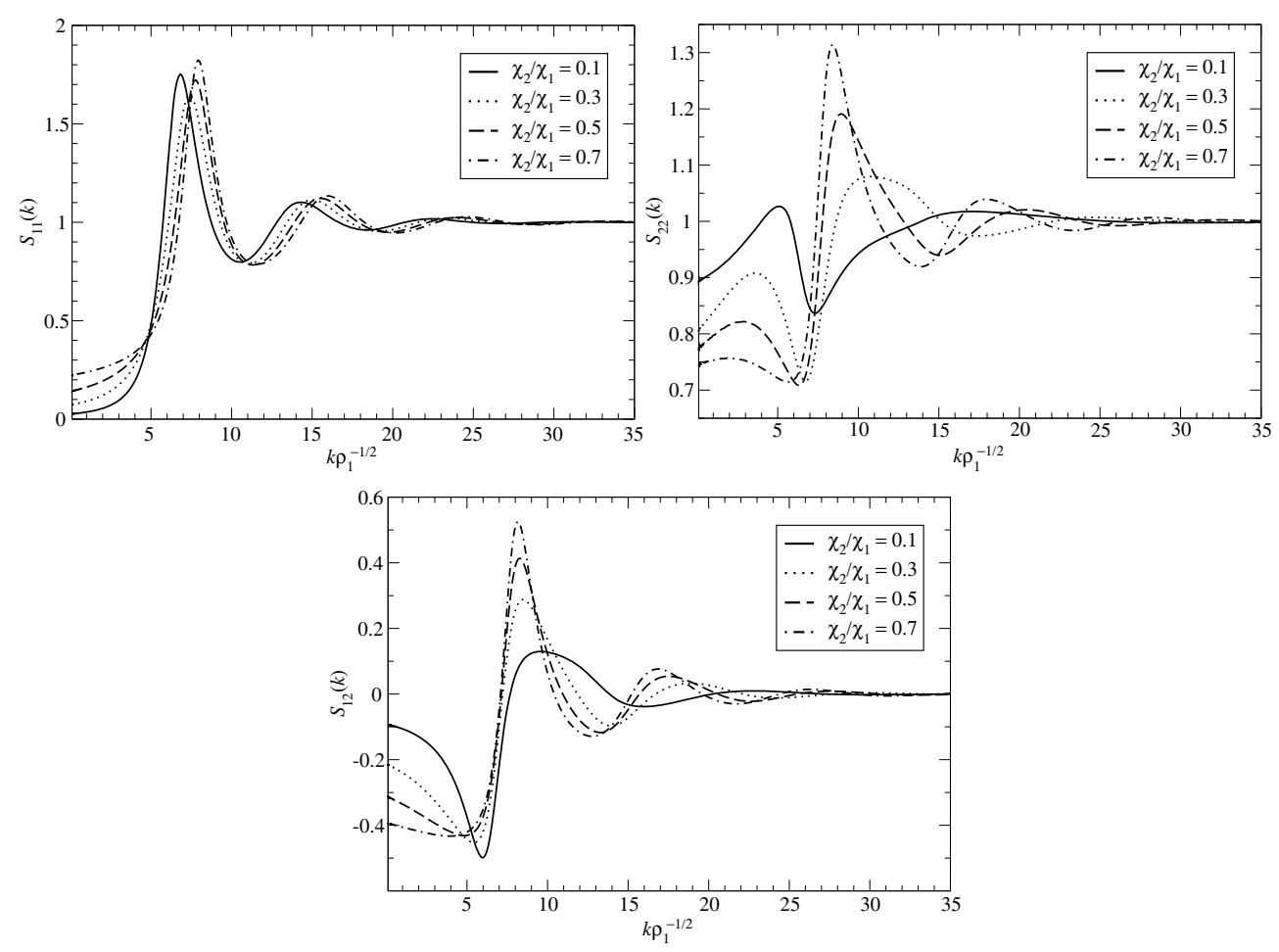

Figure 7. Partial structure factors for the two-component system and their dependence on the susceptibility ratio $\chi_{2} / \chi_{1}$. The other two parameters are fixed at $\Gamma_{11}=3.0$ and $\rho_{2} / \rho_{1}=0.5$.

a mapping onto effective hard-disc diameters $\sigma_{i j}$ is performed according to the BarkerHenderson procedure:

$$
\sigma_{i j}=\int \mathrm{d} r\left\{1-\exp \left[-u_{i j}(r) / k_{\mathrm{B}} T\right]\right\}
$$

Then, $\Delta$ is defined as

$$
\Delta=2 \sigma_{12}-\left(\sigma_{11}+\sigma_{22}\right)
$$

the same quantity that is shown in figure 8 .

The dependence of $\tilde{\Delta} \equiv \Delta /\left(\sigma_{11}+\sigma_{22}\right)$ on $\xi$ is shown in figure 8. Clearly, the case $\xi=0$ leads to $\tilde{\Delta}=-1$, whereas $\xi=1$ corresponds to $\tilde{\Delta}=0$. The negativity of $\tilde{\Delta}$ for all $\xi$-values provides an explanation for the absence of macrophase separation because it expresses the physical fact that the particles on average dislike the 'opposite' species less than they dislike their own. The negativity of $\tilde{\Delta}$ together with the property $u_{22}(r)<u_{12}(r)<u_{11}(r)$ for all $r$ lies at the heart of the subclustering phenomenon for the small particles. Indeed, by trying to create many contacts between the small particles, the system is reducing its energy due to the first part of the inequality above. At the same time, a full separation between big and small is also unfavourable, since the big particles would then have mostly contacts with each other, a feature that is disfavoured by the second part of the inequality. The system then finds the best compromise by creating irregular clusters of the small particles, which are nested in the voids formed by the big ones. As the topology and morphology of this mutual arrangement cannot 


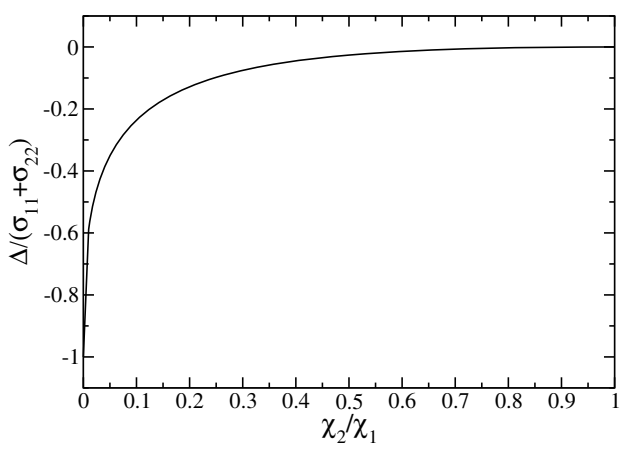

Figure 8. The non-additivity parameter $\Delta$ of the two-component magnetic mixture (equation (18)) against the susceptibility ratio $\chi_{2} / \chi_{1}$.
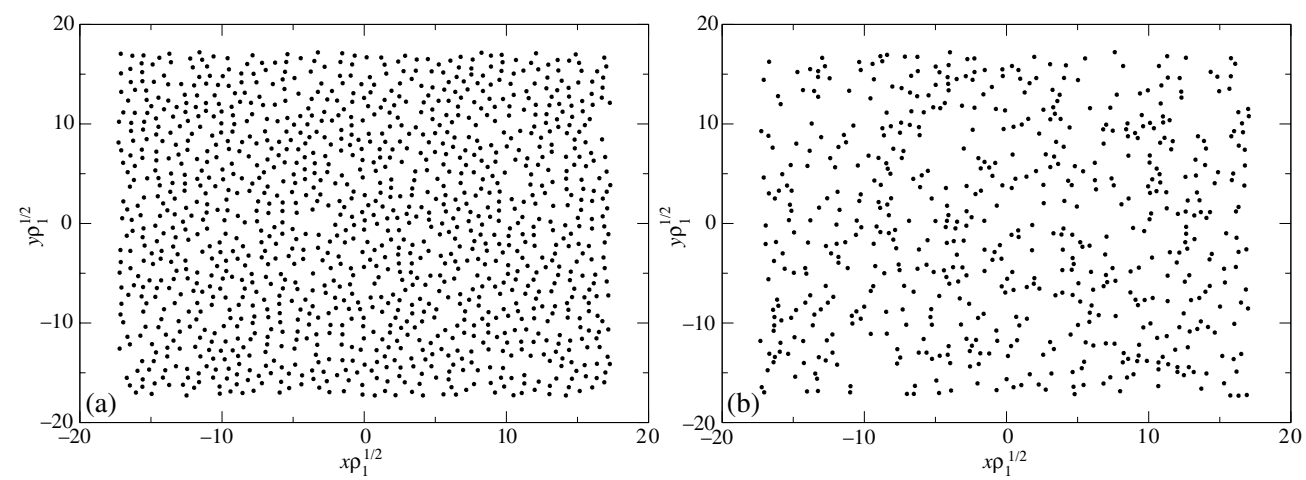

Figure 9. Simulation snapshot of the big particles (left panel) and the small particles (right panel) for the following parameters: $\Gamma_{11}=3.0, \rho_{2} / \rho_{1}=0.5, \chi_{2} / \chi_{1}=0.5$.

be unambiguously determined based on the reciprocal-space information alone, we proceed in the following section with a complementary morphological analysis in real space.

\section{Morphological analysis}

A real-space perspective of the spatial arrangements of the two components is shown in figure 9, a typical simulation snapshot of the big and small particles, respectively, for the parameter combination $\Gamma_{11}=3.0, \chi_{2} / \chi_{1}=0.5$ and $\rho_{2} / \rho_{1}=0.5$. Here, the total system contains 1200 big and 600 small particles. It can be seen that the big particles have a semi-compact arrangement: whereas in most parts of the snapshot they are close to each other, there exist also void regions in which the small particles fit perfectly. Note that the void sizes in the left part of figure 9 are not visible in reciprocal space: the only length scale featuring prominently as a peak in $S_{11}(k)$ is the one corresponding to the typical interparticle separation. Since the big particles have to make space for the voids, this scale is smaller than $a_{11}$ (which is the typical interparticle distance without small particles); hence the shift of the peak of $S_{11}(k)$ at larger $k$-values observed in figures 5 and 6.

The mutual arrangement and the nesting of the irregular clusters of small particles in the voids of the big ones is made manifest in figure 10, where we show big and small particles together. It is evident that small particles cluster in the holes formed by the 'sponge-like' 


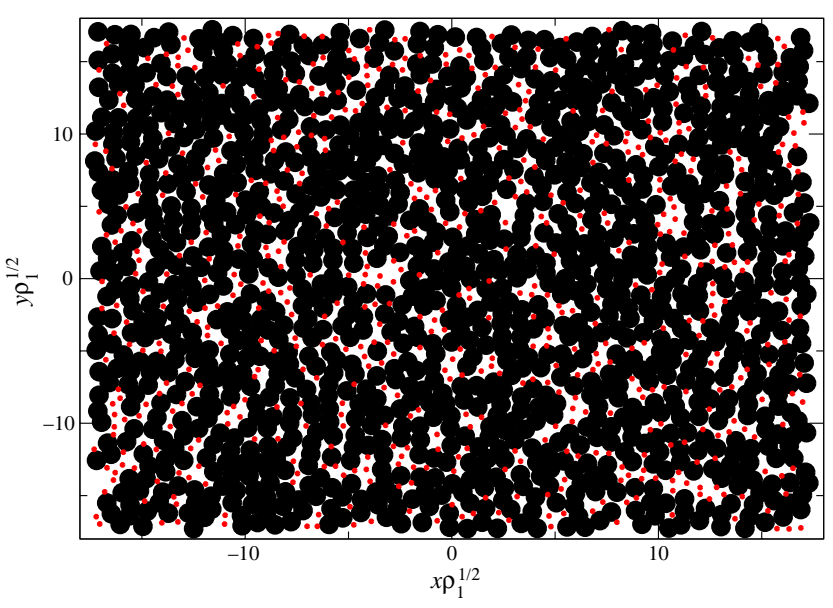

Figure 10. Simulation snapshot of big particles and small particles together, for the parameter combination of figure 9 .

(This figure is in colour only in the electronic version)

structure of the big ones. Yet, an inspection with the bare eye is dangerous and it can lead to erroneous results. It is pertinent that we quantify the morphology of the mixture, and for this purpose we employ in what follows a robust measure borrowed by integral geometry, namely a topological invariant of the pattern formed by the particles, the Euler characteristic $X[33,34]$.

The Euler characteristic is first defined for any closed, bounded, and convex set (closed body) $K$ in an $d$-dimensional space $\mathbf{E}^{d}$, with a regular boundary. We denote the set of all such bodies as $\mathcal{K}$. For such bodies, $X$ is defined as

$$
X(K)= \begin{cases}1, & \text { for convex } K \in \mathcal{K} \subset \mathbf{E}^{d}, \quad K \neq \emptyset, \\ 0, & \text { for } K=\emptyset .\end{cases}
$$

The definition can be extended to bodies with irregular boundaries (such as polygons in two dimensions) by considering the smooth $\epsilon$-parallel body, $K_{\epsilon}$, consisting of the union of $K$ and all points within a distance $\leqslant \epsilon$ from the boundary of $K$ [33]. By expanding the $d$-dimensional volume of $K_{\epsilon}$, in powers of $\epsilon$, the Euler characteristic of the irregular body is then proportional to the coefficient of the power $\epsilon^{d}$. It has precisely the same value, 1 for convex bodies and 0 for the empty set, as given by equation (19) above for bodies with a smooth boundary. Further, the definition of $X$ can be extended to non-convex shapes containing an arbitrary number of holes and belonging to the set $\mathcal{R}$ of subsets of $\mathbf{E}^{d}$ that are constructed from finite unions and intersections of convex bodies including the empty set $\emptyset$ (clearly, $\mathcal{K} \subset \mathcal{R}$ ). The extension of $X$ to such sets follows from the property of sub-additivity:

$$
X(A \cup B)=X(A)+X(B)-X(A \cap B),
$$

for any $A, B \in \mathcal{R}$. Notice that the Euler characteristic of the union of two sets $A$ and $B$ is the sum of the individual ones minus the Euler characteristic of the intersection ${ }^{6}$.

${ }^{6}$ In fact, the Euler characteristic is just one of the $d+1$ Minkowski functionals $[33,34]$ of a body embedded in $d$-dimensional Euclidean space, and all share the sub-additivity property; in three dimensions, the four Minkowski functionals are the volume, exposed area, integral mean curvature and Euler characteristic $X$; in two dimensions, they are the covered area, exposed line length and, again, $X$. For the volume, area and length the sub-additivity property is evident. 
The morphological analysis of the spatial pattern formed by the particles was carried out as follows. The particle positions of any snapshot were marked and covering circles of varying radii $R_{0}$ were superimposed on the position of each particle. The circles were filled, resulting thereby in a spatial black-and-white pattern whose appearance depends on $R_{0}$ as well as on the original particle positions. Interpreting the black parts as 'full' and the white parts as 'empty', the pattern consists, for any choice of $R_{0}$, of a number of mutually disconnected components $D\left(R_{0}\right)$ as well as a number of holes $H\left(R_{0}\right)$. The Euler characteristic $X\left(R_{0}\right)$ is an integer number and in two dimensions it is simply expressed as ${ }^{7}$ :

$$
X\left(R_{0}\right)=D\left(R_{0}\right)-H\left(R_{0}\right) .
$$

For instance, $X=1$ for a circle but $X=0$ for a ring, $X=-1$ for an eight-shaped union of two rings and so on. For the system at hand, we have at small $R_{0}$ as many mutually disconnected components as particles, $D=N$; there are no holes $(H=0)$ and hence $X / N=1$. For very large covering radii, the whole pattern is black, $D=1, H=0$ and $X / N \rightarrow 0$ for $N \rightarrow \infty$. Information on the topology of the mixture is gained by the behaviour of the $X / N$ versus $R_{0}$-curve at intermediate $R_{0}$ values.

The recognition of disconnected components and holes may appear straightforward at first yet it is a challenging problem of image analysis and processing. The problem can be simplified by digitizing the image created by the covering circles. To this end, we have imposed a fine quadratic grid on the picture, with lattice constant $l=0.05 R_{0}$. Any quadratic pixel was assigned the value 'empty' or 'full' according to the following criterion: if the centre of a pixel is closer to the centre of any circle than the radius of the latter, the pixel is 'full'; otherwise, it is 'empty'. We assign now 'Ising-like spin variables' $u(i, j)$ that live on the dual lattice, characterized by a binary 'emptiness variable', $u(i, j)=1$ if the pixel at which $u(i, j)$ is centred is empty and $u(i, j)=0$ if it is full. Here, $i, j$ are integer indices sequentially labelling the lattice sites in the horizontal and vertical directions, respectively; $i, j=1,2, \ldots, L$ in an $L \times L$ square lattice. The digitized image generates therefore a morphological lattice model and the Euler characteristic $X[u]$ of each configuration $[u]=\{u(i, j)\}, i, j=1,2, \ldots, L$, can be calculated locally and involves up to four-spin interactions only. It is given by the expression

$$
\begin{array}{r}
X[u]=\sum_{i} \sum_{j}\{-u(i, j)+u(i, j)[u(i+1, j)+u(i, j+1)] \\
-u(i, j) u(i+1, j) u(i, j+1) u(i+1, j+1)\} .
\end{array}
$$

The double sum runs over all lattice sites. Periodic boundary conditions were employed for the pixels at the borderlines of the $L \times L$-square. For a sketch of the derivation of equation (22) above, we refer the reader to appendix $\mathrm{B}$ and for a more general discussion to the appendix of [33]. The analysis was carried out for a large number of simulation snapshots and the expectation value $\langle X\rangle / N$ was calculated as a function of $R_{0}{ }^{8}$

Results of this procedure are shown in figure 11 for a particular parameter combination $\left(\Gamma_{11}=3.0 ; \chi_{2} / \chi_{1}=0.5\right.$ and $\left.\rho_{2} / \rho_{1}=0.5\right)$ for which the structural data featured a cluster prepeak at $S_{22}(k)$. The quantity shown is always $\langle X\rangle / N$, where $N$ is the number of big or small particles, depending on the curve. A useful comparison is provided by also considering the same curves drawn for a one-component system at the same $\Gamma_{11}$-value as well as for an ideal gas. Comparing the Euler characteristic curve of the small particles with that of the ideal gas,

7 We emphasize the space dimensionality because in three dimensions things are different: although $X$ remains an integer, it has there the form $X=D+C-T$, where $C$ is the number of cavities (as in a hollow sphere) and $T$ the number of tunnels (holes). Note also that our definition of $X$ differs by the one used in other contexts by a trivial factor 2 .

8 Note that the quantity $\langle X\rangle$ is extensive, a property that qualifies the latter to be used as a 'topological order parameter' in morphological models [33]. 


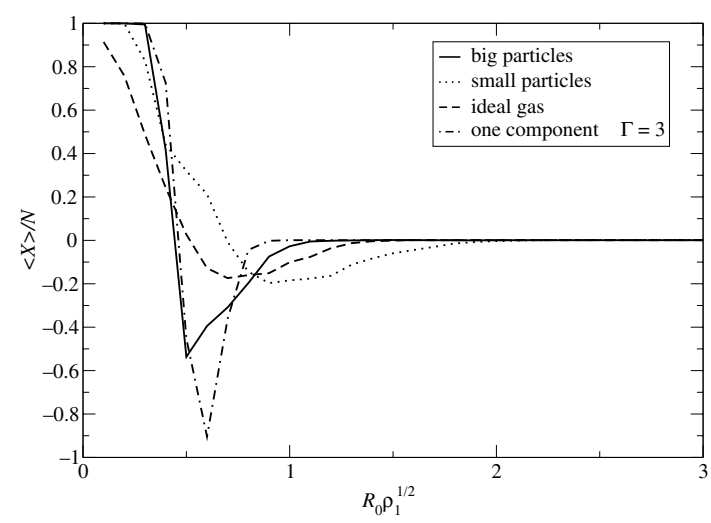

Figure 11. The expectation value of the Euler characteristic (per particle) plotted against the covering radius for a binary mixture of 1200 big and 600 small particles and for the parameters $\Gamma_{11}=3.0$ and $\chi_{2} / \chi_{1}=0.5$. The results are averaged over several simulation snapshots. For comparison, the corresponding curves of the ideal gas and a one-component system are also shown.

we see that the former decays to zero much more slowly than the latter; and the most negative part of the former occurs for larger covering radii than that of the former. Ideal particles form 'clusters' just by accident, as a result of the Poisson process of their insertion in the plane. Interacting particles form clusters in order to optimize the free energy of the mixture. The latter are thus larger and hence a larger covering radius is necessary to create holes between them.

The position of the most negative value of $\langle X\rangle / N$ for the small particles occurs at $R_{0} \sqrt{\rho_{1}} \cong 1$ and can be identified with half the typical cluster size, $\lambda_{\text {clus }} / 2$. Thus $\lambda_{\text {clus }} \sqrt{\rho_{1}} \cong 2$, a value agreeing well with the structural data extracted from figure 4 . There, the estimate $\lambda_{\text {clus }} \sqrt{\rho_{2}} \cong 1.27$ was made; thus $\lambda_{\text {clus }} \sqrt{\rho_{1}} \cong 1.8$ for the density ratio at hand. The morphological estimate is based on the consideration that, when the covering circles attain a value equal to half the cluster size, covering patches from neighbouring clusters overlap, leaving one or more holes in the space in between. And since every hole contributes -1 to the Euler characteristic, the minimum of $\langle X\rangle$ is expected for such values of $R_{0}$. Closing these holes now requires a much larger cover radius than in the ideal gas system wherein particles are more or less evenly dispersed over the whole area.

The $\langle X\rangle$ versus $R_{0}$ curves for the big particles reveal that the gaps between them are smaller than for the one-component case. This is consistent with the fact that the big particles are rearranging and open small voids in which the small ones are accommodated. In addition, the most negative value of $\langle X\rangle$ is achieved for the one-component system. This is also reasonable, since the large voids that open up for the small particles constitute a single hole and thus drive $\langle X\rangle$ less negative than the many smaller holes present in the absence of the voids. The $\langle X\rangle$ versus $R_{0}$ curves of the big and the small particles have very different shapes; there is no way to scale one on top of the other. This reveals also from the morphological point of view the very different spatial arrangements of the two species in the presence of one another.

\section{Conclusions}

We have investigated partial cluster formation in a two-component colloidal model system characterized by simple dipole-dipole interactions. The system is experimentally accessible 
and our results have been confirmed by measurements for specific parameter combinations [16]. The small-particle clusters do not form well-separated, monodisperse domains with a welldefined geometrical shape, such as those seen for interactions with a short-range attraction and a long-range repulsion [22]. They are rather diffuse, possesses a percolating spongelike topology, with a significant number of holes. We have analysed this phenomenon in terms of the non-additivity parameter of the mixture and we put forward the assertion that it should be general for all mixtures with $\Delta<0$, whose interactions are purely repulsive and additionally obey the inequalities $u_{22}(r)<u_{12}(r)<u_{11}(r)$. Future work will focus on ordered phases (alloy formation) on arrested states of such mixtures and on the effects of tilted external magnetic fields.

\section{Acknowledgments}

We thank Martin Konieczny, Dominique Levesque and Christian Mayer for helpful discussions. This work has been supported by the Deutsche Forschungsgemeinschaft (DFG) within the Collaborative Research Centre SFB-TR6, 'Physics of Colloidal Dispersions in External Fields', Project Section C3.

\section{Appendix A. Two-dimensional Fourier transforms}

In this appendix, we briefly discuss an algorithm due to Caillol et al [38], which allows for the accurate calculation of the Fourier transform (FT) in two dimensions and takes advantage of fast Fourier transform (FFT) techniques. In contrast to the three-dimensional case, the occurring integrals do not reduce to one-dimensional sine- or cosine-integral transformations, a property that calls for the implementation of special manipulations. The two-dimensional FT $\tilde{f}(k)$ of a radially symmetric function $f(r)$ takes in polar coordinates the form

$$
\tilde{f}(k)=\int_{0}^{2 \pi} \mathrm{d} \varphi \int_{0}^{\infty} \mathrm{d} r r \exp (\mathrm{i} k r \cos \varphi) f(r),
$$

with the imaginary unity i. Taking into account the integral representation of the Bessel function of zeroth order $J_{0}(z)$ as

$$
J_{0}(z)=\frac{1}{2 \pi} \int_{0}^{2 \pi} \mathrm{d} \varphi \exp (\mathrm{i} z \cos \varphi)
$$

we can rewrite equation (A.1) as

$$
\tilde{f}(k)=2 \pi \int_{0}^{\infty} \mathrm{d} r r J_{0}(k r) f(r)
$$

which defines the Hankel transform. The task of calculating convolution integrals in two spatial dimensions is thus formally reduced to calculating the Hankel transform (and its inverse, which can be easily derived using the steps above). However, equation (A.3) is not directly amenable to FFT techniques, since the integration kernel is the Bessel function $J_{0}(k r)$ and not $\sin (k r)$, as is the case in three dimensions.

To deal with this difficulty, one first introduces new variables, $R$ and $K$, that are connected to the old ones by the following relation:

$$
\begin{aligned}
& r=\exp (R), \\
& k=\exp (K) .
\end{aligned}
$$

Passing over to the new coordinates, equation (A.3) takes the form

$$
\tilde{f}(\exp (K))=\exp (-K) \int_{-\infty}^{\infty} \mathrm{d} R \exp (R) f(\exp (R)) \exp (R+K) J_{0}(\exp (R+K)) .
$$


The right-hand side of equation (A.5) can be rewritten as a convolution type integral:

$$
\tilde{f}(\exp (K))=4 \pi \exp (-K)\left\{\operatorname{Re} \int_{0}^{\infty} \mathrm{d} t \Phi(t) M(t) \exp (\mathrm{i} K t)\right\},
$$

where

$$
\Phi(t)=\frac{1}{2 \pi} \int_{-\infty}^{\infty} \mathrm{d} R \exp (R) f(\exp (R)) \exp (\mathrm{i} R t)
$$

and

$$
M(t)=\frac{1}{2 \pi} \int_{-\infty}^{\infty} \mathrm{d} R \exp (R) J_{0}(\exp (R)) \exp (-\mathrm{i} R t) .
$$

The integral $M(t)$ can be calculated analytically; see [38]. Thus, it remains to calculate $\Phi(t)$. For this purpose, an arbitrary small value $r_{m}$ is chosen, corresponding via equation (A.4) to some $R_{m}=\ln \left(r_{m}\right)<0,\left|R_{m}\right| \gg 1$, which is used to split the integral: $\int_{-\infty}^{\infty}=\int_{-\infty}^{R_{m}}+\int_{R_{m}}^{\infty}$. Changing the variable $R \rightarrow R+R_{m}$ at the second integral, we obtain

$$
\begin{aligned}
\Phi(t)=\frac{1}{2 \pi} \int_{-\infty}^{R_{m}} \mathrm{~d} R \exp (R) f(\exp (R)) \exp (\mathrm{i} R t) \\
+\frac{1}{2 \pi} \exp \left(\mathrm{i} R_{m} t\right) \int_{0}^{\infty} \mathrm{d} R \exp \left(R+R_{m}\right) f\left(\exp \left(R+R_{m}\right)\right) \exp (\mathrm{i} R t) .
\end{aligned}
$$

For sufficiently large $\left|R_{m}\right|$, we can approximate $f(r)$ in the region $\left[0, r_{m}\right]$ by a constant, $f\left(r_{m}\right)$. Therefore, the first integral on the right-hand side of equation (A.9) $\Phi_{1}(t)$ can be approximated analytically as

$$
\Phi_{1}(t)=\frac{1}{2 \pi} \exp \left(R_{m}\right) f\left(\exp \left(R_{m}\right)\right) \frac{\exp \left(\mathrm{i} R_{m} t\right)}{1+\mathrm{i} t} .
$$

The second integral on the right-hand side of equation (A.9) can be calculated by employing a standard FFT algorithm.

Summarizing: one can rewrite the Hankel transform, equation (A.1), by means of a simple coordinate transform, equation (A.4), into a convolution type integral, equation (A.6). Transforming to Fourier space, it decomposes into the product of two integrals, equations (A.7) and (A.8). One of them can be calculated analytically, whereas the other can be calculated by an FFT. Once $\Phi(t)$ and $M(t)$ are known, equation (A.6) can also be treated by FFT techniques to yield the desired Hankel transformation. In our calculations, we employed a value $r_{m}=10^{-3} a_{11}$.

\section{Appendix B. The Euler characteristic for quadratic lattices}

Consider a quadratic lattice (lattice constant $l$ ) for which some elementary plaquettes (pixels, i.e., squares of edge length $l$ ) are occupied and some are empty. The resulting spatial pattern is represented by the configuration $[u]=\{u(i, j)\}$ of the main text, with $u(i, j)=0$ (1) denoting a full (empty) site. In principle, the resulting Euler characteristic of the pattern can be calculated by employing the property of sub-additivity, equation (20). There are three types of closed sets participating in the pattern, coded as follows: 2-cells (square plaquettes), 1-cells (edges of length $l$ ) and 0-cells (points). Due to the discrete geometry, 2-cells can only intersect on 1- or 0 -cells and 1-cells only on 0-cells. We denote the Euler characteristic of a $D$-cell, $D \leqslant d=2$ ( $D$ is the topological dimension of the cell), as $X_{D}$. Evidently, it holds that $X_{D}=1$ for all three cells described above.

In calculating $X[u]$ via equation (20), track must be kept of all intersections. In order to simplify the calculation and completely avoid intersections, we now introduce the open cell $\breve{C}$ 
of any closed cell $C$, defined as $\breve{C}=C \backslash \partial C$, where $\partial C$ is the boundary of $C$. For example, every closed square can be figured as the union of the disjoint collection of its interior, its open edges and its point vertices, whereby there is no distinction between open and close points. The Euler characteristic of an open convex cell $\breve{X}_{D}$ is given by [33]

$$
\breve{X}_{D}=(-1)^{D} \text {. }
$$

Thus, $\breve{X}_{2}=1$ for an open square, $\breve{X}_{1}=-1$ for an open edge and $\breve{X}_{0}=1$ for the vertices. Since a closed square is the disjoint union of the open interior, the four open edges and the four vertices, the above considerations together with equation (20) consistently give

$$
X_{2}=1+4 \times(-1)+4 \times 1=1,
$$

whereas for a closed line segment (edge), which can be seen as the union of the open segment and the two endpoints, we again consistently obtain

$$
X_{1}=-1+2 \times 1=1 .
$$

Evidently, the computational advantage of working with open sets on a discrete lattice is that all intersections vanish and the sub-additivity of equation (20) becomes strict additivity. Let, therefore, $N_{D}[u]$ be the number of open $D$-cells present in any configuration $[u]$. Equations (20) and (B.1) above imply that the Euler characteristic $X[u]$ is a linear superposition:

$$
X[u]=\sum_{D=0}^{2} \breve{X}_{D} N_{D}[u]=N_{0}[u]-N_{1}[u]+N_{2}[u] .
$$

It is straightforward to show that the quantities $N_{D}[u]$ are given by the expressions

$$
\begin{aligned}
& N_{2}[u]=\sum_{i} \sum_{j}[1-u(i, j)] ; \\
& N_{1}[u]=\sum_{i} \sum_{j}\{2-u(i, j)[u(i+1, j)+u(i, j+1)]\} ; \\
& N_{0}[u]=\sum_{i} \sum_{j}[1-u(i, j) u(i+1, j) u(i, j+1) u(i+1, j+1)] .
\end{aligned}
$$

Equation (22) of the main text immediately follows from equations (B.4)-(B.7) above.

\section{References}

[1] Zahn K and Maret G 1999 Curr. Opin. Colloid Interface Sci. 460

[2] Löwen H 2001 J. Phys.: Condens. Matter 13 R415

[3] Zahn K, Lenke R and Maret G 1999 Phys. Rev. Lett. 822721

[4] Zahn K and Maret G 1999 Phys. Rev. Lett. 853656

[5] Wille A, Valmont F, Zahn K and Maret G 2002 Europhys. Lett. 57219

[6] Zahn K, Wille A, Maret G, Sengupta S and Nielaba P 2003 Phys. Rev. Lett. 90155506

[7] Eisenmann C, Keim P, Gasser U and Maret G 2004 J. Phys.: Condens. Matter 16 S4095

[8] Eisenmann C, Gasser U, Keim P and Maret G 2004 Phys. Rev. Lett. 93105702

[9] von Grünberg H H, Keim P, Zahn K and Maret G 2004 Phys. Rev. Lett. 93255703

[10] Froltsov V A, Blaak R, Likos C N and Löwen H 2003 Phys. Rev. E 68061406

[11] Froltsov V A, Likos C N and Löwen H 2004 J. Phys.: Condens. Matter 16 S4103

[12] Froltsov V A, Likos C N, Löwen H, Eisenmann C, Gasser U, Keim P and Maret G 2005 Phys. Rev. E 71031404

[13] van Teeffelen S, Likos C N, Hoffmann N and Löwen H 2006 Europhys. Lett. 75583

[14] Kollmann M, Hund R, Rinn B, Nägele G, Zahn K, König H, Maret G, Klein R and Dhont J K G 2002 Europhys. Lett. $\mathbf{5 8} 919$

[15] Löwen H, Messina R, Hoffmann N, Likos C N, Eisenmann C, Keim P, Gasser U, Maret G, Goldberg R and Palberg T 2005 J. Phys.: Condens. Matter 17 S3379 
[16] Hoffmann N, Ebert F, Likos C N, Löwen H and Maret G 2006 Phys. Rev. Lett. 97078301

[17] Finken R, Louis A A and Hansen J-P 2000 Phys. Rev. E 61 R1028

[18] Archer A J, Likos C N and Evans R 2002 J. Phys.: Condens. Matter 1112031 and references therein

[19] Sear R P and Gelbart W M 1999 J. Chem. Phys. 1104582

[20] Archer A J, Likos C N and Evans R 2004 J. Phys.: Condens. Matter 16 L297

[21] Malescio G and Pellicane G 2003 Nat. Mater. 297

[22] Imperio A and Reatto L 2004 J. Phys.: Condens. Matter 16 S3769

[23] Imperio A and Reatto L 2006 J. Chem. Phys. 124164712

[24] Stell G 1995 J. Stat. Phys. 78197

[25] Góźdź W T and Evans R 2003 J. Chem. Phys. 1183702

[26] Seul M and Andelman D 1995 Science 267476

[27] Stirner T and Sun J 2005 Langmuir 216636

[28] Liu Y, Chen W-R and Chen S-H 2005 J. Chem. Phys. 122044507

[29] Mladek B M, Gottwald D, Kahl G, Neumann M and Likos C N 2006 Phys. Rev. Lett. 96045701

[30] Mossa S, Sciortino F, Tartaglia P and Zaccarelli E 2004 Langmuir 2010756

[31] Sciortino F, Mossa S, Zaccarelli E and Tartaglia P 2004 Phys. Rev. Lett. 93055701

[32] Sear R P, Chung S-W, Markovich G, Gelbart W M and Heath J R 1999 Phys. Rev. E 59 R6255

[33] Likos C N, Mecke K R and Wagner H 1995 J. Chem. Phys. 1029530

[34] Mecke K R and Stoyan D 2000 Statistical Physics and Spatial Statistics, the Art of Analyzing and Modeling Spatial Structures and Pattern Formation (Springer Lecture Notes in Physics vol 554) (Berlin: Springer)

[35] Jackson J D 1975 Classical Electrodynamics (New York: Wiley)

[36] Rogers F J and Young D A 1984 Phys. Rev. A 30999

[37] Hansen J-P and McDonald I R 1986 Theory of Simple Liquids (London: Academic)

[38] Caillol J M, Levesque D and Weis J-J 1981 Mol. Phys. 44733

[39] Biben T and Hansen J-P 1991 J. Phys.: Condens. Matter 3 F65

[40] Lekner J 1991 Physica A 176485

[41] Zahn K, Méndez-Alcaraz J M and Maret G 1997 Phys. Rev. Lett. 79175

[42] Allen M P and Tildesley D J 1987 Computer Simulation of Liquids (Oxford: Clarendon)

[43] Lang A, Likos C N, Watzlawek M and Löwen H 2000 J. Phys.: Condens. Matter 125087

[44] Ashcroft N W and Langreth D C 1967 Phys. Rev. 156685

[45] Lekkerkerker H N W, Poon W C K, Pusey P N, Stroobants A and Warren P B 1992 Europhys. Lett. 20559 